TP Periodica Polytechnica Mechanical Engineering

\author{
58(1), pp. 15-25, 2014 \\ DOI:10.3311/PPme.7397 \\ Creative Commons Attribution (i)
}

RESEARCH ARTICLE

\section{Predicting the Performance of an Industrial Centrifugal Fan Incorporating Cambered Plate Impeller Blades}

\author{
Lucio Cardillo, Alessandro Corsini, Giovanni Delibra, Franco Rispoli, \\ Anthony G. Sheard, Paolo Venturini \\ RECEIVED 12 October 2013; Accepted 31 January 2014
}

\begin{abstract}
Application of computational methods to industrial fan design processes has progressed steadily over the past decade. The reducing cost of the computer hardware upon which codes run has brought the hardware within the reach of all industrial fan designers. However, the cost of commercially available codes remains high. Open source codes provide industrial fan designers with an alternative. The finite volume open-source solver OpenFOAM has been used by scholars to predict the performance of industrial centrifugal fans incorporating impeller blades constructed from cambered plate, but not by industrial fan designers. This paper presents a modelling approach which we developed for application as part of an industrial fan manufacturers order related design process. We compare numerical performance predictions with experimental results both at peak pressure and at peak efficiency conditions. As a further possible investigation, the simulated flow field is used to predict the patterns of erosion of the impeller.
\end{abstract}

\section{Keywords}

Industrial Fan - Centrifugal Fan - Fan Performance Prediction ' Erosion prediction ' Open Source Solver · Computational Fluid Dynamics

\section{Lucio Cardillo}

Alessandro Corsini email: alessandro.corsini@uniroma1.it Giovanni Delibra

Franco Rispoli

Dipartimento di Ingegneria Meccanica e Aerospaziale,

Sapienza University of Rome

Via Eudossiana 18, I-00184 Rome, Italy

Anthony G. Sheard

Fläkt Woods Limited

Axial Way, Colchester, CO4 5ZD, UK

Paolo Venturi

Dipartimento di Ingegneria Meccanica e Aerospaziale,

Sapienza University of Rome

Via Eudossiana 18, I-00184 Rome, Italy

\section{Introduction}

The industrial centrifugal fans used in cement, steel and power applications are typically tailored to the applications' specific flow and pressure demands [1]. Although aerodynamic performance has historically been scaled from previous laboratory test data, the impeller, shaft and housing mechanical design, bearing selection and rotor-dynamic analysis are invariably unique. A consequence of the need for a unique design for each fan has resulted in a historic focus on automating the processes associated with mechanical design and rotor-dynamic analysis [1]. Design methods that embed previously established limits into a computer code are coupled with parametric three-dimensional Computer Aided Design (CAD) models that have associated two-dimensional drawing packs. The result are design methods that engineers can use to deliver a full set of manufacturing drawings for a centrifugal industrial fan with less than one man week of engineering time required for each design.

A historic focus on the automation and control of industrial centrifugal fan order related engineering and production of manufacturing drawings has helped to ensure that in-service failures are rare; however, this ultimately limits the resultant design's aerodynamic efficiency. Scaling and interpolating fan performance using methods developed in the 1950's [2,3] results in reliably predicting aerodynamic performance, but inevitably also sub-optimal performance.

The European Union Regulation 327 sets minimum Fan and Motor Efficiency Grades (FMEG's) for industrial fans. The 2013 minimum FMEG's have resulted in approximately 33 per cent of fan sales before 1 January, 2013, now being illegal within Europe as a consequence of their low efficiency. The European Union will raise minimum FMEG's on 1 January, 2015. In the USA, the Department of Energy has been monitoring the regulatory activity within Europe. On 1 February, 2013, the US federal government published a framework document in the Federal Register outlining the intended approach to fan regulation that aims to eliminate inefficient industrial fans within the USA by 2019. The industrial fan community widely anticipates that the Department of Energy will adopt the same approach as the European Union, increasing the minimum allowable fan and motor efficiency within three years of introducing the initial 2019 targets. 
With today's regulatory framework it is reasonable to assume that the practice of scaling aerodynamic performance from historic test data will become progressively less acceptable. The resultant fan efficiency associated with doing so will be increasingly unlikely to meet the minimum allowable fan efficiency. Industrial fan designers will be driven to apply the same computational methods that are commonplace within the aerospace community, optimising fan performance within the mechanical constraints imposed by each specific application.

Over the last two decades, engineers have developed computational fluid dynamic techniques specifically focused on centrifugal fans. Zhang et al. [4] computed the three-dimensional viscous flow in a backwards swept centrifugal fan impeller's blade passage at its design point by solving the Reynolds Averaged Navier-Stokes (RANS) equations using standard eddy viscosity turbulence closure. Thakur et al. [5] studied a centrifugal blower and developed a quasi-steady rotor-stator modelling technique. Khelladi et al. [6] studied the impeller-diffuser unsteady interactions by using an unsteady computational method.

A reason for the difficulty associated with accurately predicting centrifugal industrial fan performance is that their impellers are typically fabricated from cambered plate. A consequence of using cambered plate impeller blades is that engineers inevitably associate impellers with regions of separated flow. This makes accurately predicting fan performance using computational methods challenging. However, recent research has focused on elucidating the flow-field features associated with interaction between the impeller and diffuser [7], interaction between the impeller-scroll-inlet ring [8] and the clearance flow impact on overall fan performance $[9,10]$. The research objective has been to systematically characterise the flow-field in poorly conditioned regions of separated flow, and provide a basis for modelling the actual flow-field.

In this paper we present a method for predicting an industrial centrifugal fan's performance when the fan impeller incorporates cambered plate blades using the unstructured opensource finite volume solver OpenFOAM $[11,12]$. We model the time-varying flow at a fan impeller-volute interface using the 'frozen rotor' approach that researchers have shown results in accurate predictions of centrifugal fan performance $[10,13]$. We describe how we connected the impeller and volute grids using an Arbitrary Mesh Interface (AMI) [14]. We conducted the research on a typical example of an industrial centrifugal fan incorporating an impeller with cambered plate blades [15].

We then explain how we verified the chosen modelling approach's effectiveness by comparing computational results with experimental results at both peak efficiency and peak pressure conditions. Agreement between the two was within the measured data's uncertainty, and consequently the computational method was effective in the application. We then explain how we analysed secondary flow structures to provide an insight into the flow-field physics.
As a demonstration of possible use of the information obtained by the described simulation approach, prediction of the flow field evolving within the impeller is then used to predict erosion, with the aim educting the patterns of those areas which are exposed the most to this detrimental phenomenon.

The paper ends with a summary of the effectiveness of OpenFOAM when used with the developed modelling approach and the resultant implications for industrial centrifugal fan design praxis.

\section{Fan Description}

The studied fan is part of a family of fans intended for process industry application with a pressure developing requirement of up to $20 \mathrm{kPa}$. The family includes impellers with backwardswept cambered plate blades that are the subject of the reported research, Table 1. A double-inlet with the inlet plenum incorporating 90 degree bends and a double impeller characterises the chosen fan, Figure 1 and Table 2. We experimentally derived the studied fan's aerodynamic performance in accordance with ISO 5801 requirements [16].

Tab. 1. Fan range description

\begin{tabular}{ll}
\hline size range, $\mathrm{D}_{2}$ & $3 \mathrm{~m}$ to $4 \mathrm{~m}$ \\
performance standard & ISO $5801-$ ISO 13347 \\
volume flow rate & up $750 \mathrm{~m}^{3} / \mathrm{s}$ \\
total pressure rise & up to $50 \mathrm{kPa}$ \\
rotational speed & $900-1000 \mathrm{rpm}$ \\
blade sections & cambered plate \\
\hline
\end{tabular}

Tab. 2. The studied fan geometry

\begin{tabular}{ll}
\hline impeller inlet diameter & $1804 \mathrm{~mm}$ \\
impeller outlet diameter & $3440 \mathrm{~mm}$ \\
volute outlet diameter & $5600 \mathrm{~mm}$ \\
impeller blade width & $400 \mathrm{~mm}$ \\
volute width & $200 \mathrm{~mm}$ \\
impeller blade count & 11 \\
rotational speed & $900 \mathrm{rpm}$ \\
\hline
\end{tabular}

\section{Modelling Approach}

An issue when modelling centrifugal fans is that the geometry does not allow for common techniques that engineers use when modelling axial turbomachinery. For example, engineers usually apply periodic boundary conditions, which simulate the flow-field in just one blade passage. When modelling a centrifugal fan it is necessary to model the entire impeller in order to account for the coupling between the impeller and the asymmetric volute casing within which it is housed. 
The 'frozen rotor' approach is a reliable and accurate modelling technique for predicting the flow-field for an entire centrifugal fan impeller when in an asymmetric volute casing $[17,18]$. The frozen rotor approach neglects the unsteady coupling between the impeller and volute casing. The impeller's position is fixed during the simulation relative to the volute casing, with the resulting computation at steady-state. We can account for the impeller motion relative to the volute casing by using a multiple frame methodology [18]. A multiple frame methodology enables one to account for the Coriolis and centrifugal forces within the impeller. We explicitly computed Coriolis forces within the Navier-Stokes momentum equation and applied centrifugal forces after we completed the steadystate computation [19].

We spatially resolved the coupling between the impeller and volute casing computational domains over the impeller's periphery using an Arbitrary Mesh Interface approach (AMI) [14]. We solved the incompressible Reynolds-Averaged NavierStokes equations using the finite volume method and a standard $\mathrm{k}-\varepsilon$ turbulence model with the synthetic wall-treatment available in OpenFOAM [17].

We carried out simulations with OpenFOAM [11,12] using the built-in solver MFRSimpleFOAM. Computations were carried out using a second order upstream interpolation for a convective kinetics divergence scheme in order to diminish the numerical diffusivity. We solved the linearised system of equations by adopting an ILU preconditioned semi-iterative conjugate gradient linear solver, in combination with a SIMPLE segregation scheme and OpenFOAM's smoothSolver for all other equations. In all cases the convergence tolerance was set to $10^{-8}$ for all the computed quantities. We performed the computations on the computer cluster Matrix of Cineca (Rome) over 48 processors. The initial fan flow-field's computation at the

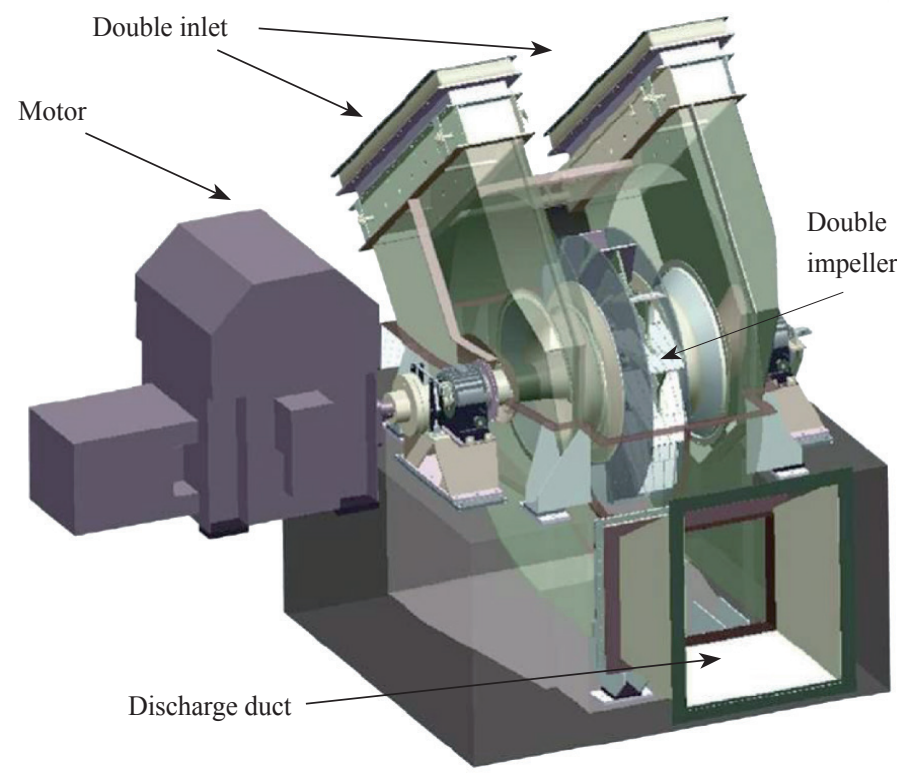

Fig. 1. An example of a centrifugal fan with double inlet and cambered plate blades from the studied family of industrial fans. peak efficiency duty point required 48 hours of computational time. We then used the peak efficiency duty point solution as a starting point for the fan flow-field's peak pressure computation that required a further eight hours of computational time.

\section{Fan geometry, boundary conditions and grid sensitivity analysis}

An impeller with both a double inlet and double impeller characterises the studied fan, Figure 1. To minimise the required computational effort for the simulation, we modelled only one half of the impeller to exploit the impeller's symmetry and effectively reduce the necessary computational effort to predict the flow-field by half. We modelled the resultant half-fan in three parts: the inlet, the impeller and the volute casing, Figure 2. These three regions were coupled using AMI interfaces.

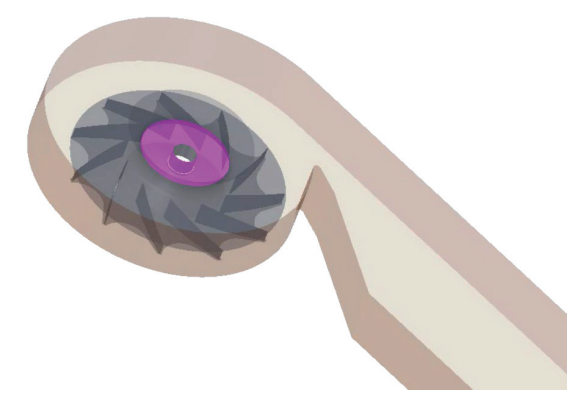

Fig. 2. The modelling technique adopted modelled one half of the double inlet - double width impeller. The computational domain was split into three parts: and inlet region (shown in purple), an impeller region (shown in black) and a volute casing region (shown in grey).

We assessed simulation sensitivity to grid density with a power coefficient as a convergence parameter, Table 3 . The comparative analysis between grids focused on the inlet and impeller blade passage grids. We increased the blade-to-blade grid density within the impeller from an initial coarse level of 50,000 cells to a fine level of 300,000 cells with both hub-toshroud and blade-to-blade refinement. The simulation results appeared unaffected by the blade-to-blade grid density, and therefore we used the coarse blade-to-blade passage grid.

Coupling the aforementioned three regions of the mesh (inlet, impeller and volute) by means of AMI interface we were able to produce a high-quality hexahedral mesh without the usual constraints of hexahedral meshes and keeping the total count of cells around $6.1 \times 10^{6}$. Details of the mesh are shown in Figure 3, while an assessment of the grid quality and resolution is given in Table 4, that provides data for mesh quality indicators (aspect ratio, minimum included angle, volume ratio, skewness, cell number) for the three different region of the grid. The same Table 4 includes $\mathrm{y}+$ values onto the solid surfaces to provide quantitative data of the mesh resolution. Exploiting the AMI technology we were able to produce a very good mesh using 
only hexahedral cells. We estimate from previous experience that an unstructured tetrahedral mesh would require approximately $20 \times 10^{6}$ cells, increasing the computational effort because of both more cells and the necessary iterations to reach a converged solution.
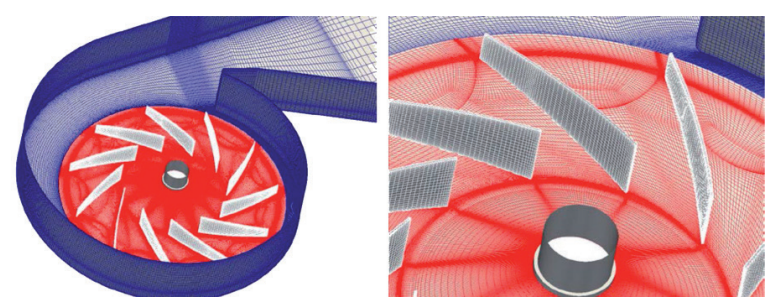

Fig. 3. Computational mesh for the studied fan; overview (left) and rotorstator interface (right).

Tab. 3. Grid sensitivity analysis

\begin{tabular}{llll}
\hline & Coarse & Fine & Exp $\phi=0.047$ \\
$\lambda$ power coeff. & 0.0451 & 0.0453 & 0.0459 \\
\hline
\end{tabular}

Tab. 4. Simulation mesh data with grid quality indicators and $y^{+}$values on solid walls

\begin{tabular}{|c|c|c|c|}
\hline & \multicolumn{3}{|c|}{ Impeller-number of cells: $3.3 \mathrm{M}$} \\
\hline & Min & Average & Max \\
\hline Aspect ratio & 1.0 & 5 & 32.8 \\
\hline Minimum included angle & 20.3 & 65.3 & 90 \\
\hline Volume ratio & 1.0 & 1.4 & 22.5 \\
\hline \multirow[t]{3}{*}{ Skewness } & 0.0 & 0.13 & 0.67 \\
\hline & \multicolumn{3}{|c|}{ Volute-number of cells: $1.0 \mathrm{M}$} \\
\hline & Min & Average & Max \\
\hline Aspect ratio & 1.0 & 5.2 & 116.7 \\
\hline Minimum included angle & 17.1 & 75.4 & 90 \\
\hline Volume ratio & 1.0 & 1.3 & 2.5 \\
\hline \multirow[t]{3}{*}{ Skewness } & 0.0 & 0.05 & 0.64 \\
\hline & \multicolumn{3}{|c|}{ Inlet - number of cells: $1.8 \mathrm{M}$} \\
\hline & Min & Average & Max \\
\hline Aspect ratio & 1.0 & 4.4 & 33.3 \\
\hline Minimum included angle & 17.6 & 76.2 & 89.7 \\
\hline Volume ratio & 1.02 & 1.47 & 6.65 \\
\hline \multirow[t]{3}{*}{ Skewness } & 0.0 & 0.06 & 0.69 \\
\hline & \multicolumn{3}{|c|}{$y^{+}$values on solid walls } \\
\hline & Min & Average & Max \\
\hline Blades & 2 & 90 & 290 \\
\hline Hub & 7 & 118 & 118 \\
\hline Shroud & 4 & 72 & 146 \\
\hline Volute & 8 & 94 & 165 \\
\hline
\end{tabular}

We set the inflow boundary conditions to mimic an inflow distortion which is produced because of the inlet plenum geometry. We derived the distorted inflow from previous simulations that included the inlet plenum that we used to complement a systematic experimental study [20]. The inflow velocity distribution illustrates the presence of a large flow distortion, with the distortion dependent on the inlet plenum geometry, Figure 4. This flow distortion occurs because of the $90 \mathrm{deg}$ bend and the limited space available in practical application, and consequently compromises the inlet plenum geometry aerodynamically. The two-dimensional velocity vectors, coloured with the axial flow coefficient, illustrate both a separated flow region and highly asymmetric flow into the impeller, Figure 4 . We adopted an average inflow turbulence level of ten per cent and set a dissipation length scale at ten per cent of mean blade chord. Standard wall function were used at the solid walls. We imposed zerogradient boundary conditions on the diffuser outflow.

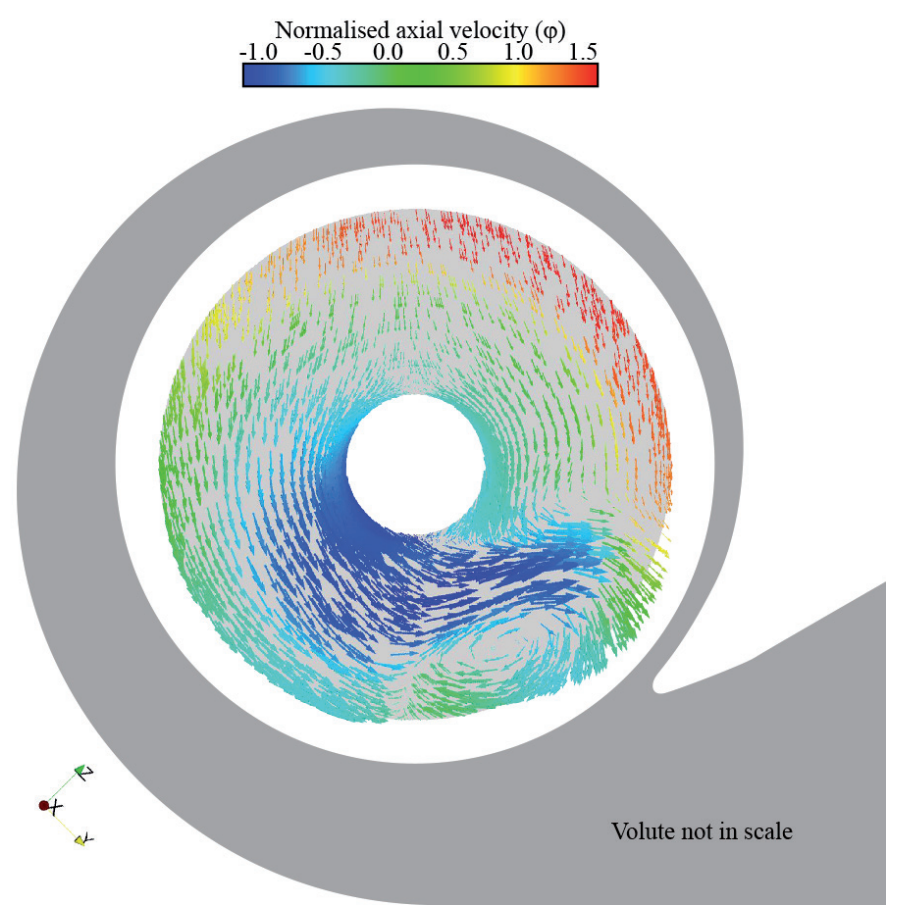

Fig. 4. Inflow velocity profiles coloured with normalised axial velocity $(\varphi)$. The volute is not shown to scale.

\section{Particle transport and dispersion, and erosion modeling}

In a Lagrangian framework, particle transport and dispersion may be performed adopting two different approaches: computing the motion of each particle (SPT, Single Particle Tracking), or modelling the behaviour of a cluster of particles, only tracking its mean path [21]. The former approach requires to solve the particle equation of motion (that is, the Basset-BoussinesqOseen - BBO - equation [22]) at run-time using the calculated flow-field parameters at the particle's current location. On the 
contrary, the latter approach involves tracking a statistical representation of some clusters of particles. Here we adopted this statistical approach, namely the Particle Cloud Tracking (PCT) model, first proposed by Baxter [23], and then developed and used by a number of researches [24-29]. Although the SPT provides more accurate results, the computational effort required is between one and two orders of magnitude larger than that required for PCT, thus for industrial applications the PCT is more suitable [28-31]. The cloud equation of motion is the ensemble averaged version of the BBO equation, reduced in accordance to the application's constraints [23]. Particle distribution within a cloud is assumed to be Gaussian, and the cloud size depends on the turbulent characteristics of the flow. Since in the original PCT each particle moves with the mean cloud velocity, which may strongly affect the erosion prediction, here we adopted a modified version of the PCT. As a first approximation we assume that the ratio between the drift and the flow velocity at the mean position of the cloud is constant within a cloud; accordingly we compute the particle local velocity at a boundary cell as:

$$
\left|\mathbf{v}_{\mathrm{bc}}\right|=\left|\mathbf{u}_{\mathrm{bc}}\right|\left(1-\frac{\left|\mathbf{v}_{\text {drifit }}\right|}{\left|\mathbf{u}_{\mathrm{m}}\right|}\right)
$$

where $\mathbf{u}_{\mathrm{bc}}, \mathbf{v}_{\mathrm{bc}}$ are respectively the flow and particle velocity vectors in the centre of the boundary cell, $\mathbf{v}_{\text {drift }}=\mathbf{u}_{\mathrm{m}}-\mathbf{v}_{\mathrm{m}}$ is the drift velocity vector at the cloud's centre, $\mathbf{u}_{\mathrm{m}}$ and $\mathbf{v}_{\mathrm{m}}$ are the flow's and cloud's velocity vectors (respectively) at the cloud centre. Further details about the PCT model can be found in [23], [28-31], [32].

Erosion is a very complex phenomenon depending on several factors such as impact velocity, impact angle, particle shape, and material properties of both the particles and the target surface. For this reason the most realistic erosion models are semi-empirical. One of these models is the one reported by Tabackoff et al. [33] which predicts the erosion per unit mass of impacting particles $(E R$, in $\mathrm{mg} / \mathrm{g})$ of different materials:

being

$$
E R=K_{1} f_{\alpha}\left|\mathbf{v}_{b c}\right|^{2} \cos ^{2} \alpha\left(1-R_{T}^{2}\right)+f_{i}
$$

$$
\begin{aligned}
& R_{T}=1-0.0061\left|\mathbf{v}_{b c}\right| \sin \alpha \\
& f_{\alpha}=\left\{1+K_{C}\left[K_{12} \sin \left(90 \alpha / \alpha_{0}\right)\right]\right\}^{2} \\
& f_{i}=K_{2}\left(\left|\mathbf{v}_{b c}\right| \sin \alpha\right)^{4}
\end{aligned}
$$

where $\alpha$ is the impact angle, $K_{1}, K_{2}, K_{12}$ and $K_{C}$ are functions of material properties of particles and target surface. The authors used Tabackoff's [33] model in this study, assuming that the centrifugal fan is made of stainless steel. Therefore, according to Tabackoff, material coefficients used are reported in Table 5, and the angle of maximum erosion, $\alpha_{0}$, is equal to 30 degrees.
Tab. 5. Erosion model coefficients [33].

\begin{tabular}{ccc}
\hline$K_{C}$ & $\alpha \leq 3 \alpha_{0}$ & 1 \\
\hline & $\alpha>3 \alpha_{0}$ & 0 \\
\hline$K_{1}$ & & $1.505101 \cdot 10^{-06}$ \\
\hline$K_{12}$ & & $2.96077 \cdot 10^{-01}$ \\
\hline$K_{2}$ & & $5.0 \cdot 10^{-12}$ \\
\hline
\end{tabular}

\section{Results}

\section{Flow field}

The reported research focuses on the development and validation of a modelling approach that will enable the reliable and routine use of the open source solver OpenFOAM to predict industrial centrifugal fan performance with cambered plate impeller blades. End users of the studied class of industrial fan generally specify a design point and a test-block point. Engineers typically select the design point close to the fans' peak efficiency operating point to minimise fan running costs. They generally select the test-block point close to the fans' peak pressure operating point to minimise fan size for a given application in order to minimise capital cost. Therefore, the authors predicted the studied fan's performance at two operating points, peak pressure (PP) corresponding to a volume flow rate of $110 \mathrm{~m}^{3} / \mathrm{s}$ and the peak efficiency (PE) corresponding to a volume flow rate of $221 \mathrm{~m}^{3} / \mathrm{s}$.

The authors selected the peak pressure operating point at the very peak of the studied fan characteristic, therefore approaching stall. In practical application fan designers would not select this class of fan with a peak pressure operating point at the fan characteristic's peak. They would use a margin on pressure and would choose the peak pressure operating point at a higher flow rate than that which the authors adopted in the reported research. The authors selected the peak pressure operating point at the fan characteristic's very peak to predict fan performance when stall is incipient. This is a demanding task. Consequently, any modelling approach that can predict fan performance when stall is incipient is inherently robust.

We first established the studied fan's performance experimentally in accordance with ISO 5801 requirements [16], Figure 5. The developed modelling approach was able to predict fan performance well, Figure 5. We under predicted the calculated peak pressure operating point total pressure by 0.6 per cent and the peak efficiency operating point by 1.5 per cent. We over predicted the calculated peak pressure operating point efficiency by 3.0 per cent and the peak efficiency operating point by 1.0 per cent, Table 5 . We estimated the measured data's uncertainty as $+/-1.0$ per cent.

Although we predicted the calculated peak pressure's total pressure within the measured data's uncertainty, we under predicted both the peak pressure and peak efficiency operating point total 
pressures. The under prediction appeared to be correlated with volume flow rate. This trend of under predicting total pressure is probably a consequence of the adopted modelling approach. The adopted modelling approach neglected the unsteady interaction between the impeller and volute. It is this interaction that generates artificial diffusivity, and therefore the adopted computational grid, numerical method and turbulence model comprised did not include the necessary mechanisms to model artificial diffusivity. Artificial diffusivity plays a role in the calculation of the complex flow-field associated with centrifugal fan impellers.

Despite under predicting the total pressure, we considered the prediction of the peak pressure operating point's efficiency was in good agreement with the measured data. We selected the peak pressure operating point at the very peak of the fan's characteristic where stall is incipient. The ability of the developed modelling approach to predict total pressure and efficiency to within 1.5 per cent and 3.0 per cent respectively when stall is incipient confirms the effectiveness of the adopted modelling approach. The modelling approach was able to adequately compute the circumferential blade-fluid interaction and the resultant torque on the impeller without accounting for the unsteady impeller-volute interaction. Consequently, we may consider the adopted modelling approach as both robust enough to enable production of a converged solution when stall is incipient, and as providing a reasonable degree of accuracy with minimal computational effort.

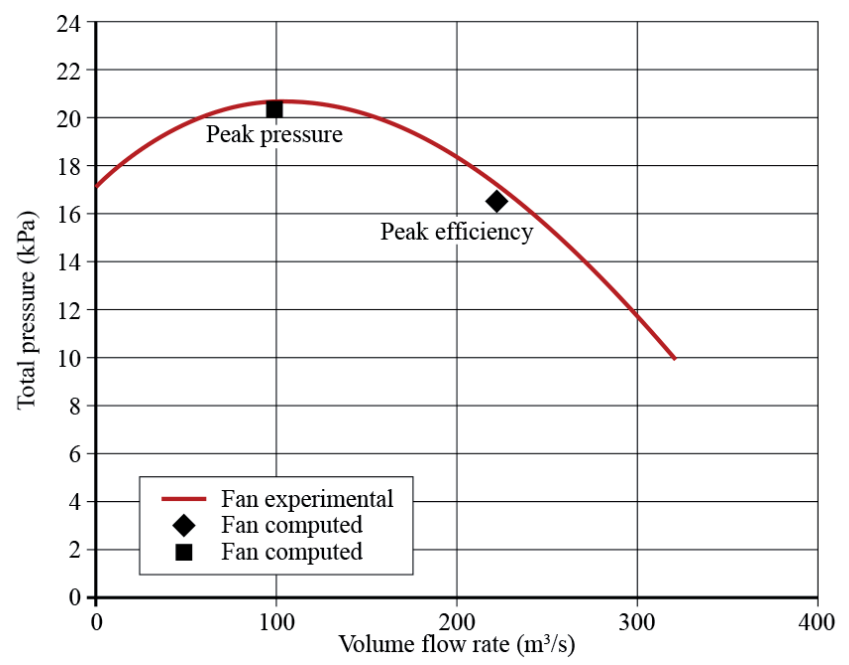

Fig. 5. Pressure-volume characteristic curve for the studied fan

Tab. 6. A comparison of experimental and computed total pressure rise and efficiency

\begin{tabular}{cccccccc}
\hline \multirow{2}{*}{ Duty point } & $\mathbf{Q}\left[\mathrm{m}^{3} / \mathrm{s}\right]$ & \multicolumn{3}{c}{$\boldsymbol{\Delta} \boldsymbol{p}_{\text {tot }}[\mathrm{kPa}]$} & & $\boldsymbol{\eta}_{\text {tot }}$ & \\
\hline & & $E X P$ & $C F D$ & $\Delta$ & $E X P$ & $C F D$ & $\Delta$ \\
PP & 110 & 20.78 & 20.46 & $-1.5 \%$ & $75 \%$ & $78 \%$ & $+3 \%$ \\
PE & 221 & 17.00 & 16.89 & $-0.6 \%$ & $82 \%$ & $81 \%$ & $-1 \%$ \\
\hline
\end{tabular}

We may assess the adopted modelling approach's effectiveness by considering the relative velocity streamlines though the impeller. The relative velocity streamlines provide an insight into the flow field's three-dimensionality around the impeller. To aid in interpreting the data, we have labelled each impeller blade, Figure 6. We show the relative velocity streamlines for both the peak pressure and peak efficiency operating points, Figure 7.

The relative velocity streamlines at the peak efficiency operating condition indicate the presence of a leading edge separation from blades B1 to B5, immediately after the junction of the blade and shroud. The blade shroud junction has resulted in the flow's non-optimal alignment onto the blades' leading edge which then results in a separated flow region within each bladeto-blade passage. In contrast, the relative velocity streamlines at the peak pressure operating condition indicate that the fan flow-field is adapting to the reduced flow-rate that occurs with incipient stall. The relative velocity streamlines indicate that separated flow is present between most of the impeller blades. The only blade-to-blade passage not affected by the separation occurring in other passages is the passage between impeller blades B1 and B2. The relative velocity streamlines indicate that all other blade-to-blade passages are either partly stalled with the blade passage between blade B10 and B11 having a double separation and is therefore completely stalled.

Relative velocity magnitude may provide a further insight into the flow-field's complexity though the studied fan. To aid in interpreting the data, we have defined two impeller reference sections, Figure 8 . We show the relative velocity magnitudes for both the peak pressure and peak efficiency operating points, Figure 9.

The relative velocity magnitude contours at the peak pressure operating condition indicate that each impeller blade-to-blade passage is associated with separated flow. The only exception is the passage between blade B1 and B2 immediately after the volute throat. The double separation in the blade passage B11

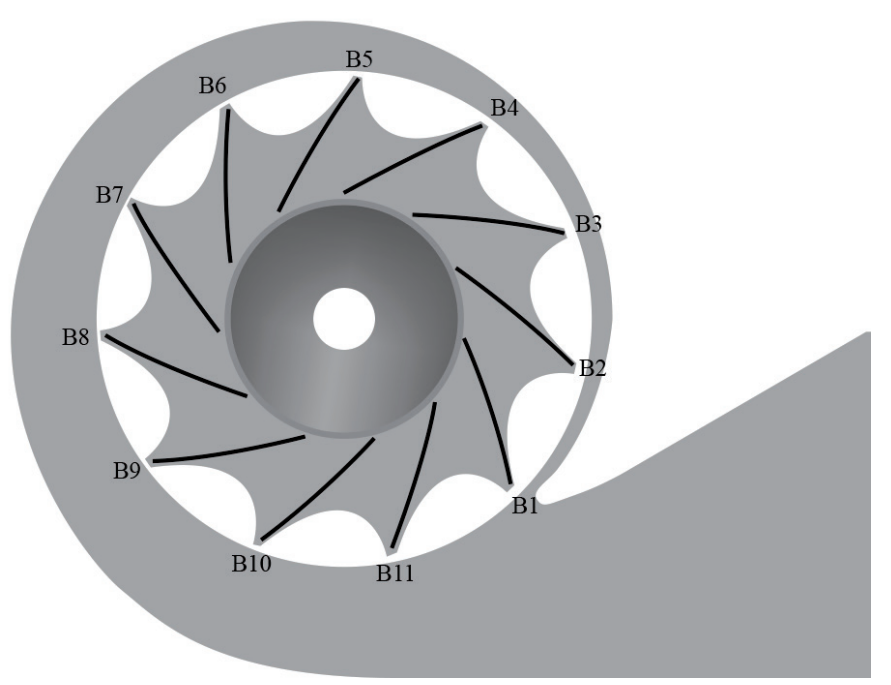

Fig. 6. Blade reference labels. 
to B1 is clearly evident, with a double separation also establishing itself in the blade passage B10 to B11. The relative velocity magnitude contours indicate that the extent of the separated flow is greater at the reference section $\mathrm{S} 1$ than $\mathrm{S} 2$. This is to be expected as the reference section S1 is closer to the impeller's back-plate and therefore the boundary layer flow on the backplate more heavily influences it.

In contrast to the peak pressure operating condition, we do not associate the peak efficiency operating condition with relative velocity magnitudes that indicate the presence of separated flow. When we studied the relative velocity magnitudes for blades B1 to B5, there was some evidence of recirculation at the blade's leading edge. This recirculation may be indicative of a non-optimal blade leading edge profile, a problem that is difficult to address when using cambered plate blades.
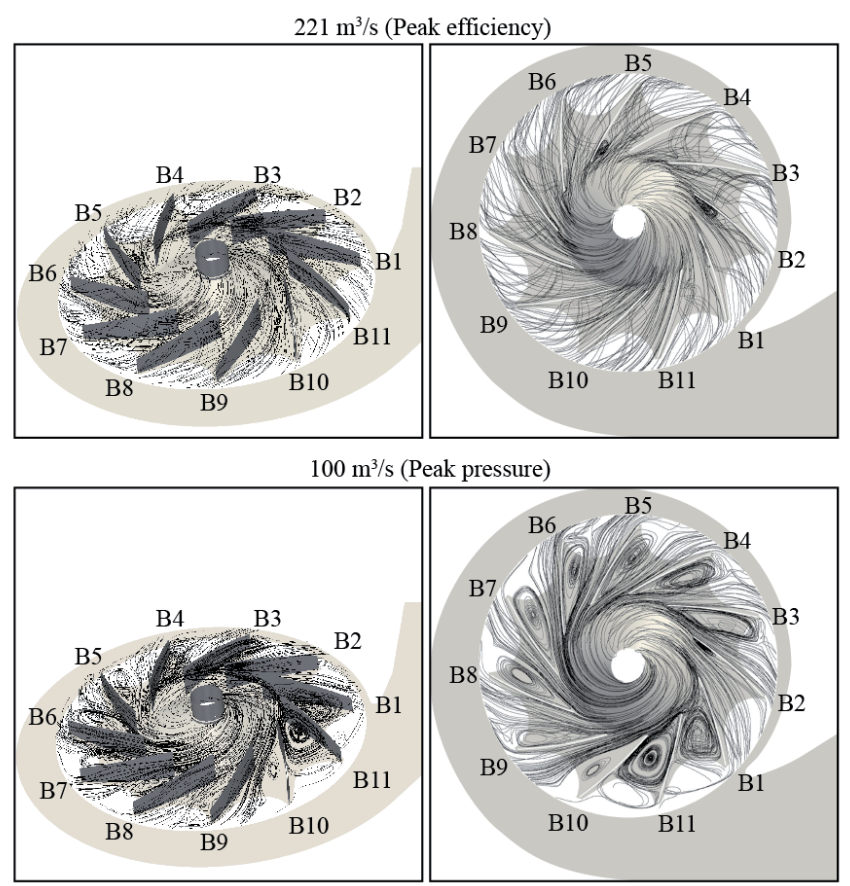

Fig. 7. Three dimensional relative streamlines through the impeller for Peak Efficiency (top) and Peak Pressure (bottom).

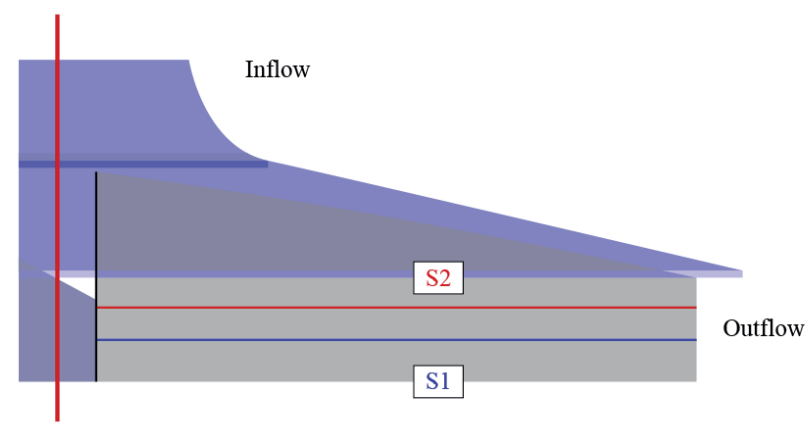

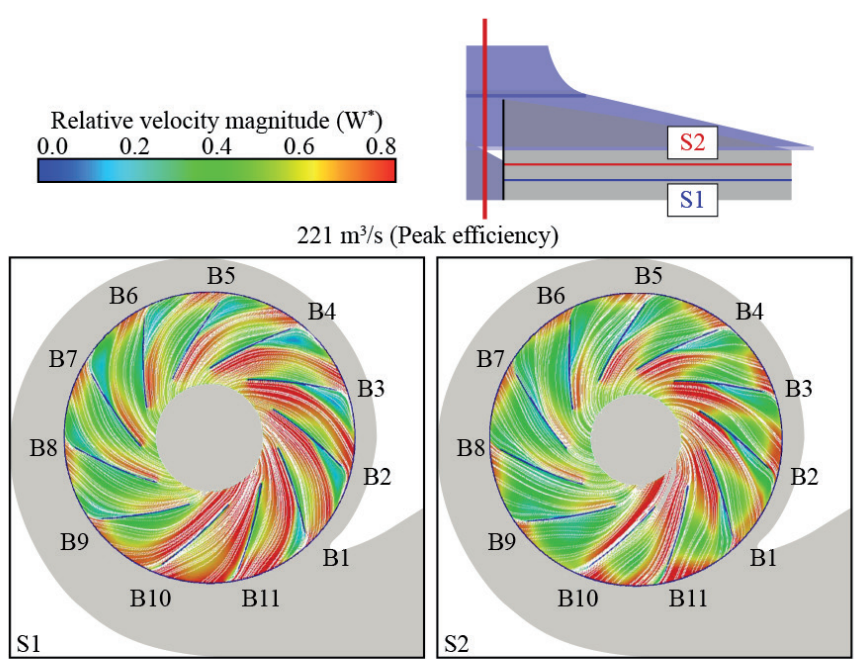

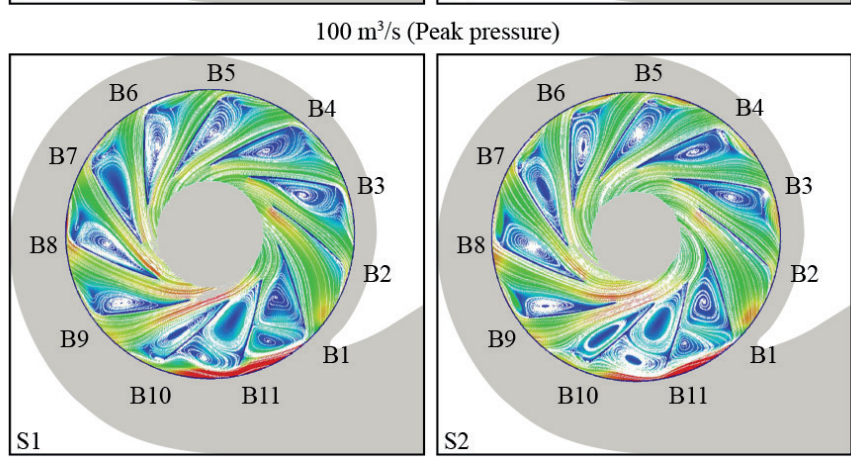

Fig. 9. Contours of the relative velocity magnitude ( $\left.\mathrm{W}^{*}\right)$ through the impeller with streamlines for Peak Efficiency (top) and Peak Pressure (bottom).

We may achieve a final assessment of the adopted modelling approach's effectiveness by considering the secondary flow structure in the discharge volute. The secondary flow structures provide an insight into the flow-field's three-dimensionality leaving the impeller, Figure 10. The secondary flow structures for both the peak pressure and peak efficiency operating points indicate that the flow through the discharge volute is highly three-dimensional. At the peak pressure operating condition the flow is able to remain attached through the diffuser, with a relatively weak secondary flow system establishing itself through the discharge volute. In contrast, a relatively stronger secondary flow characterises the peak efficiency operating condition and features through both the diffuser and the entire discharge volute. A separated flow region that establishes itself through the diffuser is clearly evident, indicating that the diffuser angle may be larger than optimal at the peak efficiency operating condition.

Fig. 8. Impeller reference sections. 


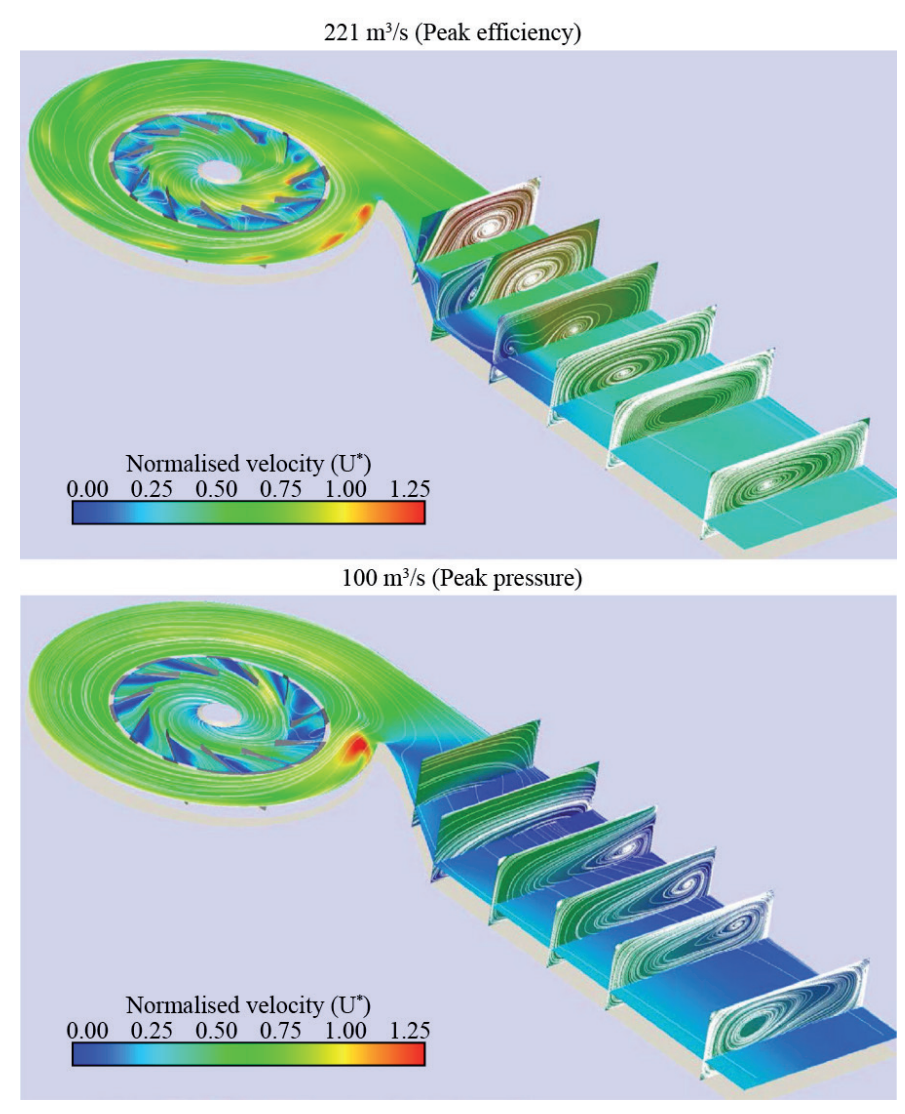

Fig. 10. Visualisation of secondary motions in the discharge duct for Peak Efficiency (top) and Peak Pressure (bottom).

\section{Particle erosion}

In order to study the erosion process we consider only the impeller, which is the component most exposed to erosion. Simulation was performed considering the flow field corresponding to a volume flow rate of $221 \mathrm{~m}^{3} / \mathrm{s}$ (so called peak efficiency or PE condition). 55 clouds, each containing $50 \cdot 10^{6}$ spherical ( $5 \mu \mathrm{m}$ size), non-rotationg and non-reacting particles, entered the domain from the inlet section of the impeller (see Figure 11). Main characteristics of the simulated particles are reported in Table 7.

Tab. 7. Main characteristics of the simulated particles.

\begin{tabular}{ccc}
\hline$\rho_{p}$ & particle diameter & initial velocity \\
\hline $2000 \mathrm{~kg} / \mathrm{m}^{3}$ & $5 \mu \mathrm{m}$ & local flow velocity \\
\hline
\end{tabular}

Cloud paths (namely the mean position as a function of time) are shown in Figure 12. Clouds enter a blade passage transported by the flow and approach the leading edge of both the blades limiting the passage (Figure 12, red ellipses). Then the combined effect of centrifugal force (due to the rotation of the impeller) and secondary flows (consequence of the blade geometry) affects the cloud motion. As a result, moving radially toward the leading edge of the blades cloud trajectories are deflected. Due to this deflection clouds approach the pressure side of the trailing edge, thus particles impact especially this region of the blade surface and possibly erode it (Figure 12, blue-dashed ellipse).

Normalized erosion rate (ER), namely the erosion rate computed according to eq. (2) and normalized with its maximum value, are illustrated in Figure 13 and Figure 14. In particular, Figure 13 shows the normalized ER patterns on the top and bottom disks of the impeller, while Figure 14 gives a view of the ER isolines within the blade passages. It is evident that erosion is not uniform, especially on the bottom disk where erosion occurs on the conical surface opposite to the inflow section, and in the transition region between the bottom wall and the blades, where the presence of two walls affects the local fluid velocity. It is worth noting that although the domain is rotating, the ER is not symmetric on the blades because of the non-symmetric flow within the impeller due to the impeller-volute interaction. This non-uniform ER is also confirmed by Figure 14 which shows the ER within the blade passages. This figure also shows that ER is larger on the leading and trailing edge regions of the suction side, and on the middle of the pressure side.

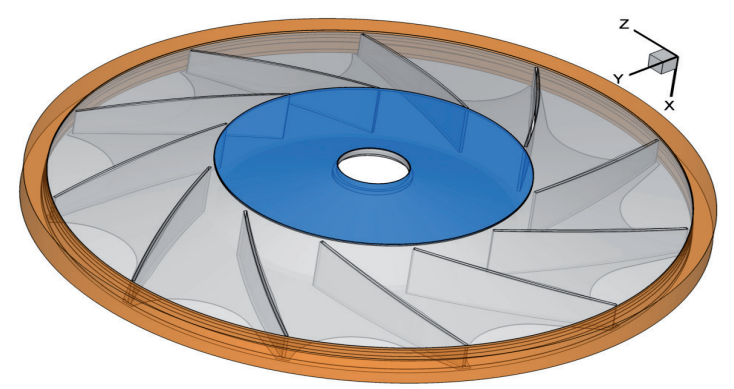

Fig. 11. Impeller: inlet (blue) and outlet (orange) surfaces.

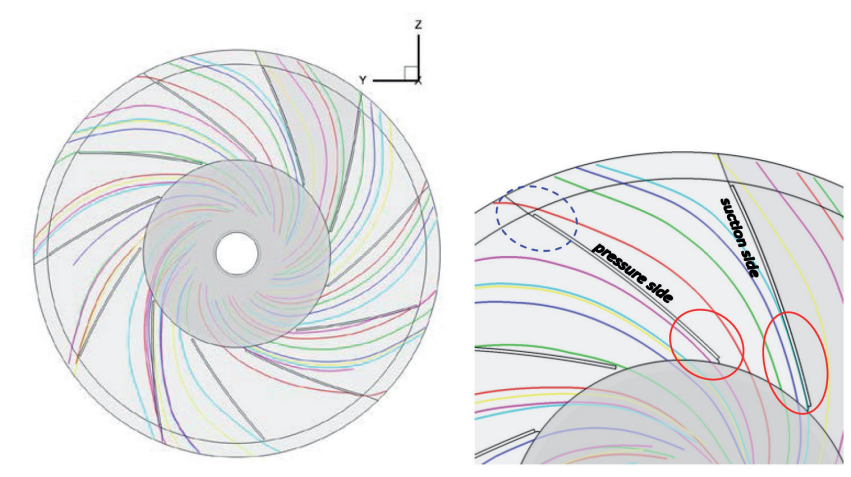

Fig. 12. Cloud trajectories used in the present simulation (left), and blade passage details (right: in red and blue ellipses the zones where trajectories are closer to leading and trailing edges, respectively). 

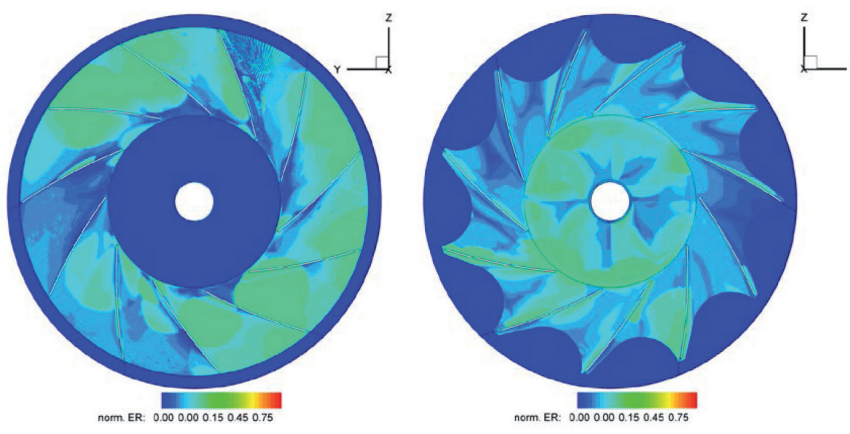

Fig. 13. Normalised erosion rate: top (left) and bottom (right) views.

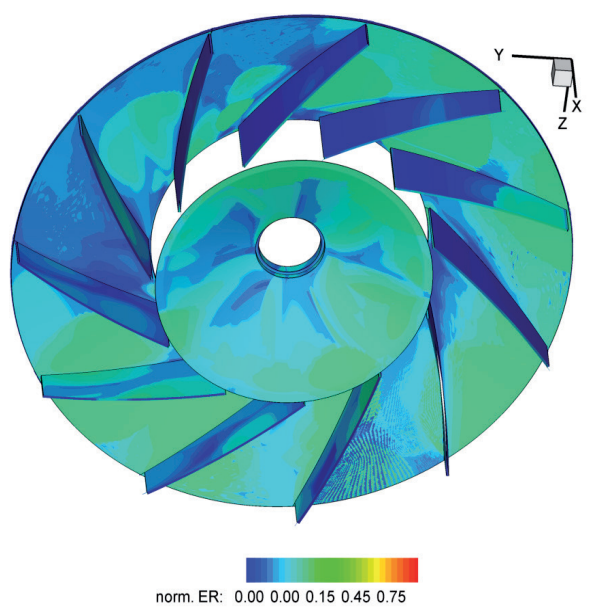

Fig. 14. Normalised erosion rate: blade view.

\section{Conclusions}

The use of open source software is an alternative to commercially available software that has the potential to provide industrial fan manufacturers with a cost effective mean of optimising the performance of industrial centrifugal fans that incorporate cambered impeller blades. The reported research in this paper presents the development and validation of a modelling approach that we implemented in the open source solver OpenFOAM. The modelling approach characterises the studied fan using a 'frozen' rotor approach and an arbitrary mesh interface. The developed modelling approach predicted the studied fan's pressure rise and efficiency reasonably accurately at the peak pressure condition when stall is incipient. This success occurred despite the frozen rotor modelling assumption that neglected unsteady interaction between the impeller and its volute casing and the use of a basic turbulence model. An analysis of the flow-field within the impeller, volute casing and discharge duct indicated that the modelling approach provides a credible prediction of the fan's secondary flow-field. Secondary flow structure predictions are in spite of the presence of extensive separated flow regions, particularly at the peak pressure operating condition.
The success of the developed modelling approach when implemented in the open source solver Open FOAM provides industrial fan manufacturers with a cost effective method for optimising industrial centrifugal fan performance when the fan incorporates and impeller with cambered plate blades. The regulatory environment facing industrial fan manufacturers has made minimum fan and motor efficiency grades a legally binding requirement within Europe, and will do so in the USA by 2019. This regulatory environment will result in the majority of industrial centrifugal fans with cambered plate impeller blades not meeting planning minimum efficiency levels, and therefore it is imperative that industrial fan designers optimise the performance of their portfolio of industrial centrifugal fans that incorporate impellers with cambered plate blades. The low cost, robustness and accuracy of the developed modelling approach when implemented in the open source solver OpenFOAM provides a practical way for industrial fan designers to engage in the optimisation process.

Results provided can be also used for further investigations such as the erosion behaviour of the fan. Erosion model can predict the regions more exposed to this phenomenon. The simulations performed put in evidence, although at this point only qualitatively, that $5 \mu \mathrm{m}$ particles mostly erode the top wall and the bottom conical wall of the impeller. Moreover erosion affects the leading and trailing edges on the suction side of the blade, as well as the central zone on the suction side.

The comprehensive approach to the erosion prediction, although still needing an optimization, is able to give useful information on the zones where erosion is more severe, and can be taken as a starting point to study new geometries for the fan minimizing the erosive effect of the particles entrained by the flow.

\begin{tabular}{|lll|}
\hline \multicolumn{2}{|l}{ Nomenclature } & \\
$D_{t i p}$ & {$[\mathrm{~m}]$} & Tip blade diameter \\
$p$ & {$[\mathrm{~Pa}]$} & Pressure \\
$p_{t o t}$ & {$[\mathrm{~Pa}]$} & Total pressure \\
$\mathrm{U}, \mathrm{W}$ & {$[\mathrm{m} / \mathrm{s}]$} & Absolute and relative velocity \\
$U_{t i p}$ & {$[\mathrm{~m} / \mathrm{s}]$} & Tip blade velocity \\
$\mathrm{K}$ & {$\left[\mathrm{m}^{2} / \mathrm{s}^{2}\right]$} & Turbulent kinetic energy \\
$\mathrm{y}^{+}$ & {$[-]$} & Normalised wall-distance \\
$\varepsilon$ & {$\left[\mathrm{m}^{2} / \mathrm{s}^{3}\right]$} & Dissipation of k \\
$\omega$ & {$[1 / \mathrm{s}]$} & Rotational speed \\
$\varphi$ & {$[-]$} & Normalised axial velocity, $\varphi=\mathrm{U}_{\text {ax }} / \mathrm{U}_{\text {bulk }}$ \\
$\eta_{\text {tot }}$ & {$[-]$} & Efficiency based on total pressure rise \\
$*$ & & Quantity normalised with $U_{t i p}$ and $D_{t i p}$ \\
\hline
\end{tabular}




\section{Acknowledgements}

The authors wish to express their gratitude to Fläkt Solyvent-Ventec, Meyzieu, France. Moreover, the authors thank Cineca for the computational resources that it made available on the Matrix cluster under the standard grant STD12-116.

\section{References}

1 Godichon A. F. E., Sheard A. G., Customer-oriented design: Flakt Solyvent-Ventec describes its e-Technopal program that is used as a fan design tool. International Cement Review, 95-99 (October 2009).

2 Daly B. B., Woods Practical Guide to Fan Engineering. Woods of Colchester Limited, Colchester, UK (1978).

3 Eck B., Fans: Design and Operation of Centrifugal, Axial-flow, and Cross-flow Fans. Pergamon Press Ltd, Oxford, UK (1973).

4 Zhang M. J., Pomfret M. J., Wong C. M., Three-dimensional viscous flow simulation in a backswept centrifugal impeller at the design point. Computers and Fluids, 25(5), 497-507 (1996). DOI: $10.1016 / 0045-7930(96) 00008-4$

5 Thakur S., Lin W., Wright J., Prediction of flow in centrifugal blower using quasi-steady rotor-stator models. Journal of Engineering Mechanics, 128(10), 1039-1049 (2002). DOI: 10.1061/(ASCE)0733-9399(2002)128:10(1039)

6 Khelladi S., Kouidri S., Bakir F., Rey R., Flow study in the impeller-diffuser interface of a vaned centrifugal fan. Journal of Fluids Engineering, 127(3), 495-502 (2005).

DOI: $10.1115 / 1.1900138$

7 Vasudeva Karanth K., Yagnesh Sharma N., CFD analysis on the effect of radial gap on impeller-diffuser flow interaction as well as on the flow characteristics of a centrifugal fan. International Journal of Rotating Machinery, 2009(Article ID 293508), 8 pages (2009). DOI: $10.1155 / 2009 / 293508$

8 Zhao Y., Song L., Wenqi H., Weixiong W., Dongtao H., Zhichi Z., Numerical simulation of flow field for a whole centrifugal fan and analysis of the effects of blade inlet angle and impeller gap. HVAC\&R Research, 11(2), 263-283 (2005). DOI: $10.1080 / 10789669.2005 .10391137$

9 Lee Y.-T., Impact of fan gap flow on the centrifugal impeller aerodynamics. Journal of Fluids Engineering, 132(9), 1-7 (2010). DOI: $\underline{10.1115 / 1.4002450}$

10 Lee Y.-T., Ahuja V., Hosagandi A., Slipper M. E., Mulvihill L. P., Birkbeck R., Coleman R. M., CImpeller design of a centrifugal fan with blade optimization. International Journal of Rotating Machinery, 2011(Article ID 537824), 16 pages (2011). DOI: $10.1155 / 2011 / 537824$

11 Weller H. G., Tabor G., Jasak H., Fureby C., A tensorial approach to continuum mechanics using object-oriented techniques. Computers in Physics, 12, 620-631 (1998).

DOI: $10.1063 / 1.168744$

12 Jasak H., OpenFOAM: a year in review. in 'Proceeding 5th OpenFOAM Workshop. Gothenburg, Sweden', 1-22 (2010).

13 Seo S.-J., Kim K.-Y., Kang S.-H., Calculations of three-dimensional viscous flow in a multi-blade centrifugal fan by modelling blade forces. Proceedings of the Institution of Mechanical Engineers, Part A: Journal of Power and Energy, 217(3), 287-297 (2003). DOI: $10.1243 / 095765003322066510$

14 Petit O., Page M., Beaudoin M., Nilsson H., The ERCOFTAC centrifugal pump OpenFOAM case study. in 'Proceeding 3rd IAHR International Meeting of the Workgroup on Cavitation and Dynamic Problems in Hydraulic Machinery and Systems. Brno, Czech Republic', 1-10 (2009).
15 Fläkt Woods Group, Solyvent product-Europe. Product catalogue, www.flaktwoods.fr

16 ISO 5801: Industrial Fans-Performance Testing using the Standardised Airways (2007).

17 Launder B. E., Sharma B. I., Application of the energy-dissipation model of turbulence to the calculation of flow near a spinning disc. Letters in Heat and Mass Transfer, 1(2), 131-137 (1974). DOI: $\underline{10.1016 / 0094-4548(74) 90150-7}$

18 Ferziger J., Peric M., Computational Methods for Fluid Dynamics. Springer (1997). DOI: $10.1007 / 978-3-642-97651-3$

19 Denton J. D., Dawes W. N., Computational fluid dynamics for turbomachinery design. Proceedings of the Institution of Mechanical Engineers, Part C: Journal of Mechanical Engineering Science, 213(2), 107-124 (1999). DOI: $10.1243 / 0954406991522211$

20 Greenzweig J., Okeley P., Korellis S., The effect of inlet flow profile distortion on fan performance. Electric Power Research Institute (EPRI), Palo Alto, California, USA, Product ID: 1020738 (2010).

21 Corsini A., Delibra G., Sheard A. G., A Critical Review of Computational Methods and Their Application in Industrial Fan Design. International Scholarly Research Network, Mechanical Engineering, 2013(Article ID 625175), 20 pages (2013). DOI: $\underline{10.1155 / 2013 / 625175}$

22 Sommerfeld M., van Wachem B., Oliemans R., Best Practice Guidelines for Computational Fluid Dynamics of Dispersed Multiphase Flows. ERCOFTAC (European Research Community on Flow, Turbulence and Combustion) Special Interest Group on Dispersed Turbulent Multi-Phase Flow (2007).

23 Baxter L. L., Turbulent transport of particles. PhD thesis, Brigham Young University, Provo, Utah, USA (1989).

24 Wang L. P., On the dispersion of heavy particles by turbulent motion. $\mathrm{PhD}$ thesis, Washington State University, Pullman, Washington, USA (1990).

25 Litchford R. J., Jeng S.-M., Efficient statistical transport model for turbulent particle dispersion in sprays. JAIAA Journal, 29(9), 14431451 (1991). DOI: $\underline{10.2514 / 3.59965}$

26 Jain S., Three-dimensional simulation of turbulent particle dispersion. PhD thesis, University of Utah, Salt Lake City, Utah, USA (1995).

27 Kær S. K., Numerical investigation of ash deposition in straw-fired furnaces. PhD thesis, Aalborg University, Aalborg, Denmark (2001).

28 Borello D., Venturini P., Rispoli F., Saavedra G. Z. R., Prediction of multiphase combustion and ash deposition within a biomass furnace. Applied Energy, 101, 413-422 (2013).

29 Corsini A., Rispoli F., Sheard A. G., Venturini P., Numerical simulation of coal fy-ash erosion in an induced draft fan. ASME Journal of Fluids Engineering, 135(8), paper no. 081303, 12 pages (2013). DOI: $10.1115 / 1.4024127$ 
30 Corsini A., Marchegiani A., Rispoli F., Venturini P., Sheard A. G., Predicting blade leading edge erosion in an axial induced draft fan. ASME Journal of Engineering for Gas Turbines and Power, 134(4), paper no. 042601, 9 pages (2012). DOI: $\underline{10.1115 / 1.4004724}$

31 Venturini P., Borello D., lossa C. V., Lentini D., Rispoli F., Modelling of multiphase combustion and deposit formation in a biomass-fed boiler: Energy, 35(7), 3008-3021 (2010). DOI: $10.1016 /$ j.energy.2010.03.038
32 Venturini P., Modelling of particle wall-deposition in two phase gassolid flows. PhD. thesis, Sapienza University of Rome, Rome, Italy (2010).

33 Tabakoff W., Kotwal R., Hamed A., Erosion study of different materials affected by coal ash particles. Wear, 52(1), 161-173 (1979). DOI: $10.1016 / 0043-1648(79) 90206-0$ 